\title{
Design of a water and oil separation tower with SA-RT and UML methodologies
}

\author{
David Alan de Oliveira Ferreira ${ }^{1}$, Fabiano da Silva ${ }^{1}$
} ${ }^{1}$ Programa de Pós-Graduação em Engenharia Elétrica da Faculdade de Tecnologia da Universidade Federal do Amazonas (FT-UFAM).
Av. General Rodrigo Octavio Jordão Ramos, 1200. Coroado I. Manaus - Amazonas - Brasil. CVEP: 69067-005.

Email:ferreirad08@gmail.com, fabianofds@bol.com.br

Received: January $28^{\text {th }}, 2017$

Accepted: February $23^{\text {th }}, 2017$

Published: March 30th 2017

Copyright $(2016$ by authors and Institute of Technology Galileo of Amazon(ITEGAM) This work is licensed under the Creative Commons Attribution International License (CC BY 4.0).

http://creativecommons.org/licenses/by/4.0/ (c) (1) (2) Open Acee:

\section{ABSTRACT}

This article discusses the design of a system that contributes to the solution of the growth of water pollution. It was developed with the main objective of designing a system of automatic separation of water and oil, elements mixed in the same reservoir, observing the analysis of the concepts of density of the fluids. The theoretical concepts were applied and the diagrams of the SA-RT and UML methodologies were illustrated. The methodologies presented are addressed in the discipline of Project Methodology and Automation Systems Programming.

Keywords: Water, oil, density, automatic separation.

\section{Projeto de uma torre de separação de água e óleo com abordagem de metodologias SA-RT e UML}

\begin{abstract}
RESUMO
Este artigo aborda o projeto de um sistema que contribui para a solução do crescimento da poluição das águas. Foi desenvolvido com o principal objetivo de projetar um sistema de separação automática de água e óleo, elementos misturados no mesmo reservatório, observando a análise dos conceitos de densidade dos fluídos. Foram aplicados os conceitos teóricos e ilustrado os diagramas das metodologias SA-RT e UML. As metodologias apresentadas são abordadas na disciplina de Metodologia de Projeto e Programação de Sistemas de Automação.
\end{abstract}

Palavras Chaves: Água, óleo, densidade, separação automática.

\section{INTRODUÇÃO}

A necessidade de implementar maneiras de preservação da natureza deve ser preocupação de todos os setores da sociedade, em residências, nos comércios e principalmente no setor industrial. A poluição do ar e das águas é a grande preocupação e deve gerar pesquisas e estudos de novas tecnologias para o tratamento de gases poluentes e resíduos que possam ser lançados em rios.

A torre de separação pode ser utilizada em aeroportos, instalações para lavagem e manutenção de veículos, ferrovias, estacionamentos e áreas de circulação intensa de veículos, instalações militares e armazenamento de petróleo e similares.

As misturas heterogêneas são misturas entre duas ou mais substâncias que não reagem entre si, apresentando assim mais de uma fase (região onde é possível enxergar o encontro dos elementos). Para realizar a separação dos elementos em uma mistura é necessário que se tenha conhecimento prévio dos mesmos, o que
Irá auxiliar na escolha do método de separação. Existem várias formas de separação dessas misturas: o processo de decantação, a sifonação e o processo que utiliza funil de bromo ou balão de decantação. O funil de bromo é um dos instrumentos utilizados em laboratório para separar uma mistura líquida heterogênea com a ação da gravidade. O elemento mais denso é drenado por uma válvula que possui um regulador de vazão no fundo do funil [1].

Este trabalha consiste na construção de um experimento científico baseado na ideia do funil de bromo e empregado para realizar a separação de dois líquidos, água e óleo, aplicando o método de separação por diferença de densidades.

Durante a execução e os testes do experimento, são aplicados conceitos teóricos pertinentes à disciplina de Metodologia de Projeto e Programação de Sistemas de Automação do curso de Pós-Graduação em Engenharia Elétrica, apresentando o croqui do projeto, lista de materiais empregados, diagramas das metodologias SA-RT e UML. 


\section{REVISÃO BIBLIOGRÁFICA}

A água é de grande importância para a vida na terra. Possui muitas propriedades para as necessidades do ser humano. Os maiores impactos ambientais resultam da contaminação hídrica dos esgotos domésticos e industriais. As preocupações com a preservação do meio ambiente tem se tornado mundialmente conhecidas, e muitos projetos e trabalhos de conscientização são desenvolvidos, tudo para o conhecimento e controle da recuperação dos ecossistemas terrestres e aquáticos [2].

A medição direta da densidade dos fluídos pode ser realizada através de sua definição padrão, ou seja, a medida de sua massa dividida pelo seu volume, conforme equação (1):

$$
\rho=\frac{m}{v} \mathrm{~g} / \mathrm{cm}^{3}
$$

A decantação é a técnica primária mais utilizada para promover a separação de água e óleo em refinarias e trata-se de um processo simples e físico. Para a separação mais minuciosa se necessita da implantação de um sistema secundário [5].

A separação utilizando o funil de bromo é uma das boas técnicas de separação de fluídos. Um exemplo da utilização deste instrumento é abordado no trabalho de obtenção de biodiesel propriamente dito [6], onde o autor utilizou a técnica após obter uma mistura heterogênea com duas fases.

\section{MATERIAIS E MÉTODOS}

O croqui apresentado na Fig. 1 representa o diagrama de montagem do protótipo de um sistema automático. Foi construído baseado na ideia do funil de bromo também conhecido como balão de decantação.

Para aplicação neste projeto foram adotados valores de densidades já conhecidos através da literatura. Aplicando-se assim os valores de $0,853 \mathrm{~g} / \mathrm{cm}^{3}$ para densidade da água e $0,9117 \mathrm{~g} / \mathrm{cm}^{3}$ para densidade do óleo [3][4].

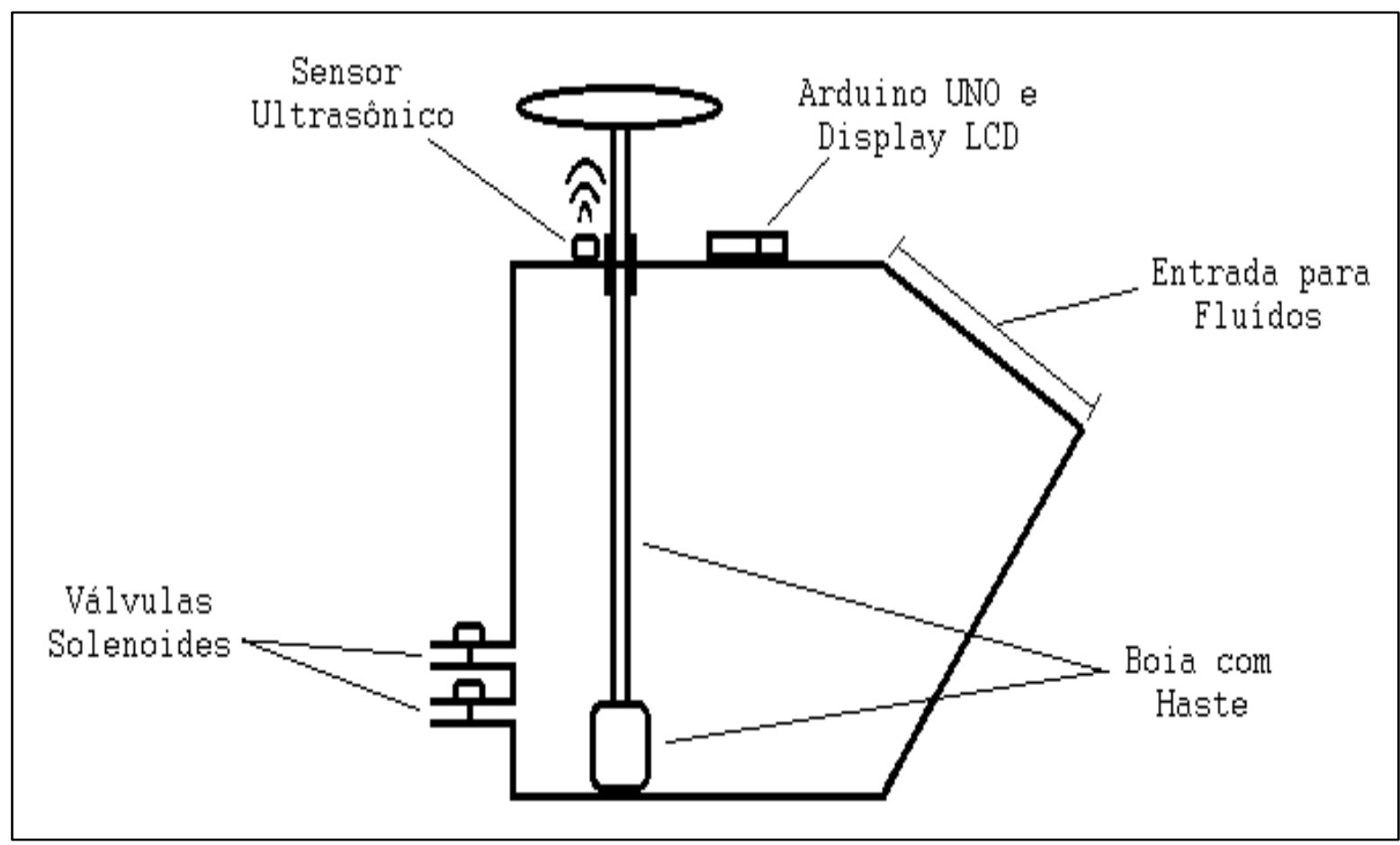

Figura 1: Croqui do Projeto.

Os principais componentes empregados no projeto são:

\section{III.1 RESERVATÓRIO}

Para construção do protótipo de estudo foi optado pelo reservatório da Figura 2. O reservatório possui dimensões:
$23 \times 41 \times 29 \mathrm{~cm}$ e capacidade de 13 litros. Optou-se por este reservatório devido ao baixo custo e pelas facilidades para adaptação no protótipo de estudo. Sua tampa está dividida em duas partes. $\mathrm{Na}$ parte plana será fixado os equipamentos que fazem parte do sistema e na parte com inclinação será a entrada para os líquidos. 


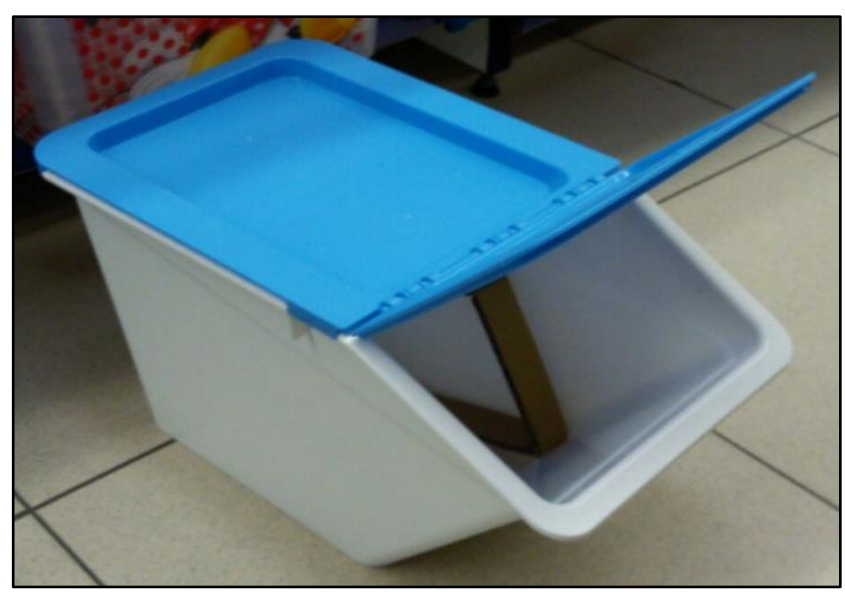

Figura 2: Reservatório.

\section{III.2 BOIA E HASTE}

Foi empregado na construção da boia na Figura 3 um pote plástico com tampa de rosca, com objetivo de preencher o interior da boia com óleo, tornando a boia de mesma densidade do óleo, o que deverá auxiliar no processo de separação dos fluídos. A haste da boia foi construída com cano de PVC, sendo prolongado para fora do tanque e fixado um disco na ponta da haste que auxiliará na medição do deslocamento da haste.

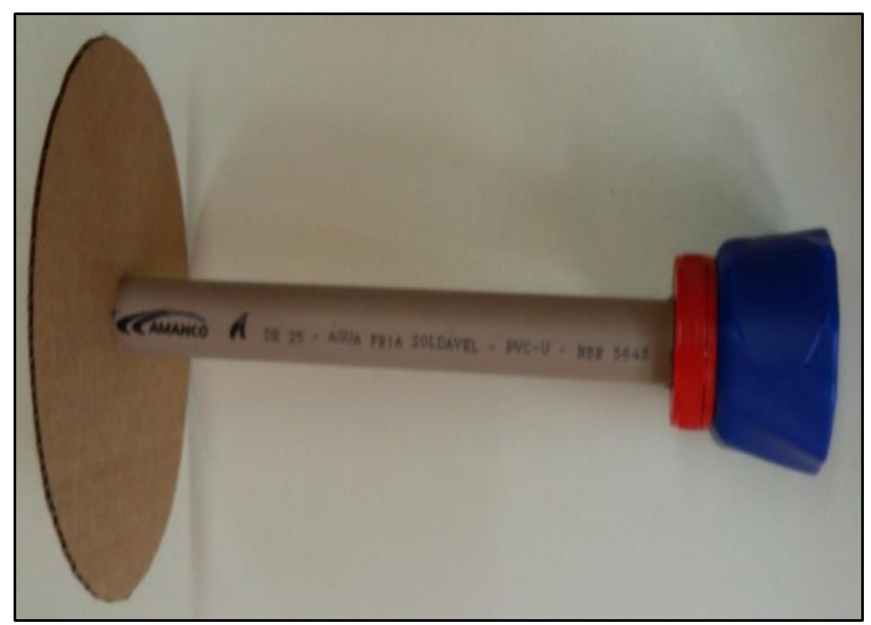

Figura 3: Boia e Haste.

\section{III.3 VÁLVULA SOLENÓIDE VA-03}

A válvula solenoide VA03 ilustrada na Fig. 4 é aplicada em automação industrial e residencial, em irrigadores automáticos, máquinas de lavar e outras aplicações. Foi escolhida esta válvula devido a seu baixo custo e facilidade de adaptação ao projeto. Abaixo temos algumas características fornecidas pelo fabricante [7].

- Tensão da bobina de 127 VAC.

- Diâmetro da conexão de $3 / 4$ de polegada.

- Possui 1 entrada e 1 saída com Angulo de $180^{\circ}$.

- Temperatura máxima do líquido é de $60^{\circ} \mathrm{C}$.

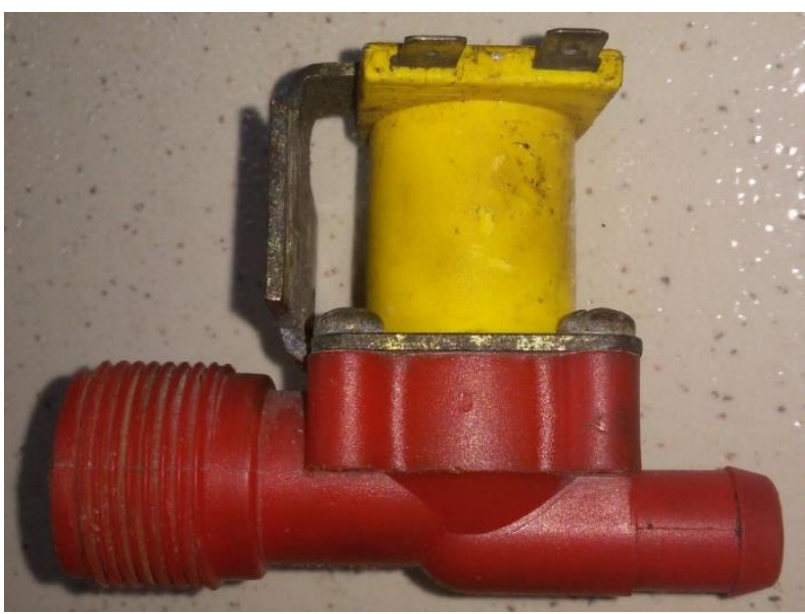

Figura 4: Válvula Solenóide.

\section{III.4 ARDUINO UNO}

O Arduino UNO, ilustrado na Figura 5, é uma placa de microcontrolador baseado no Atmega328. É largamente utilizado em trabalhos didáticos e deve ser reprojetado em um produto final. O Arduino está sendo aplicado no projeto devido ao seu baixo custo e fácil linguagem de programação. A seguir algumas características que são fornecidas pelo fabricante [8]:

- Tensão de operação 5 VCD.

- 14 pinos digitais (6 saídas PWM).

- 6 pinos de entrada analógica.

- Memória Flash de 32KB.

- Velocidade de clock de $16 \mathrm{MHz}$

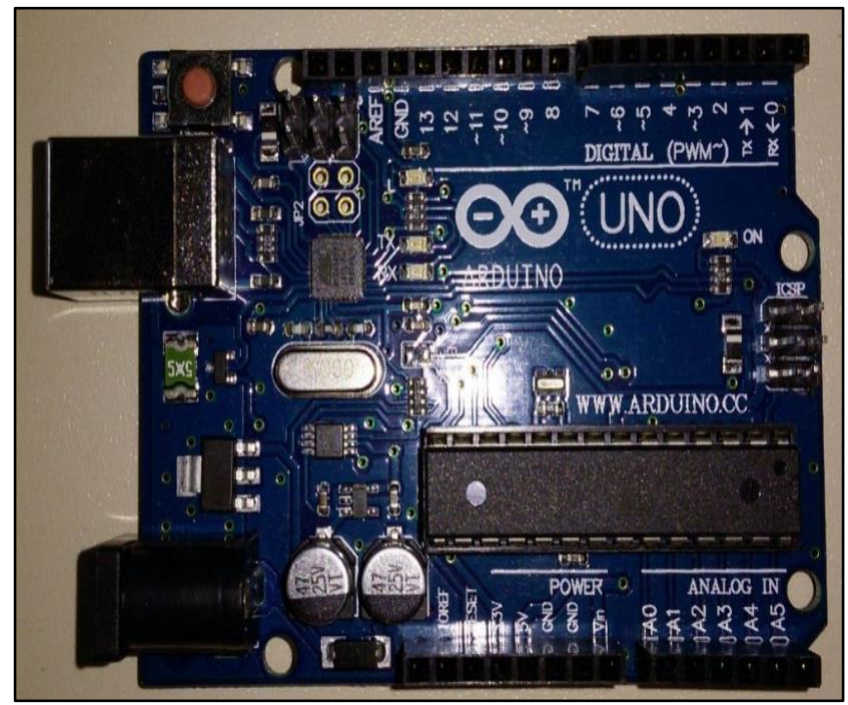

Figura 5: Arduino UNO.

\section{III.5 SENSOR ULTRASSÔNICO HC-SR04}

Sensor comum em projetos com Arduino, permite a leitura de distâncias entre $2 \mathrm{~cm}$ e 4 metros, com precisão de $3 \mathrm{~mm}$. O sensor HC-SR04 está representado na Figura 6. 


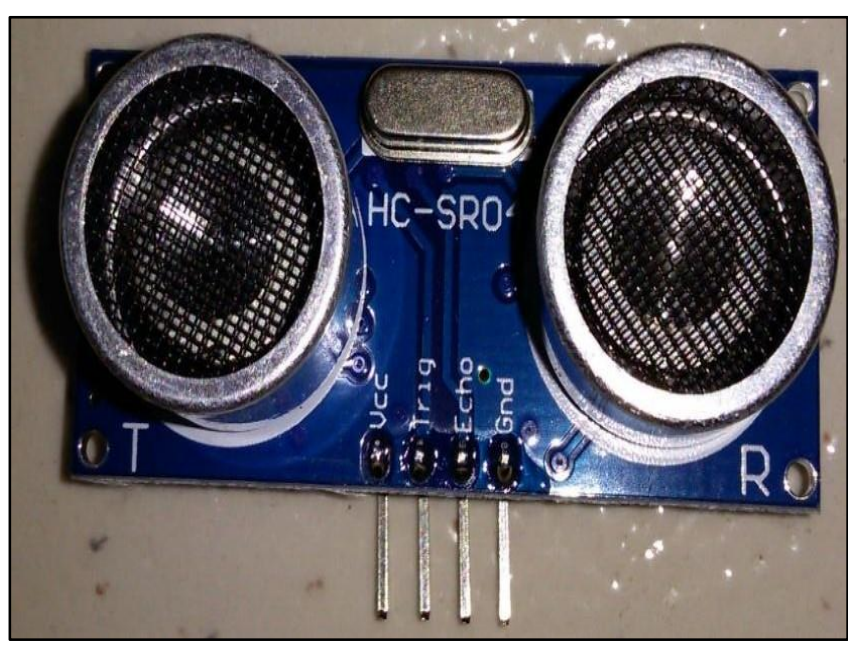

Figura 6: Sensor Ultrassônico.

\section{III.6 DISPLAY LCD}

O Display LCD é utilizado para mostrar as operações que estão sendo executadas no sistema.

- Tela $16 \times 2$.

- Cor backlight: Azul.

- Cor escrita: Branco.

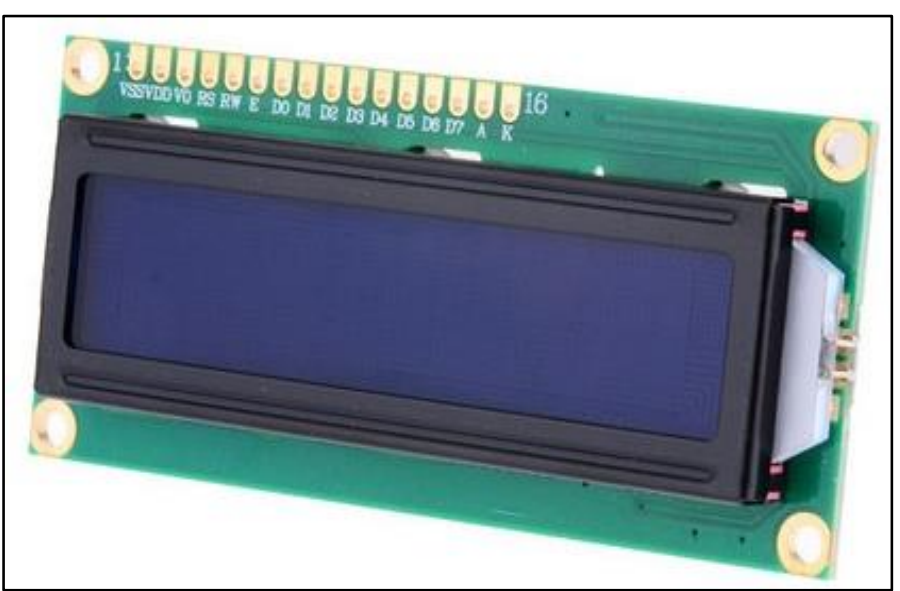

Figura 7: Display LCD.

Fonte: filipeflop.com.(2016).

\section{FUNCIONAMENTO DO SISTEMA E METODOLOGIA SA- RT}

O princípio de funcionamento de separação dos fluídos é baseado na separação por densidade dos mesmos. Os fluidos após despejados no tanque separam-se por possuírem densidades diferentes, ficando o óleo na parte superior do tanque e a água na parte inferior. A boia foi preenchida com óleo obtendo a mesma densidade do óleo de forma a se posicionar no encontro dos dois fluídos, é responsável pela movimentação da haste que é monitorada constantemente pelo sensor ultrassônico. As medições realizadas pelo sensor são enviadas ao Arduino que é responsável pelo processamento das medições e acionamento das válvulas solenoides, que são responsáveis pelo escoamento dos fluídos em recipientes. A figura 8 apresenta a montagem do sistema de separação de fluídos que foi proposto.
A metodologia SA-RT se preocupa em analisar o processo de comportamento do sistema em funcionamento. Aborda em diagramas o fluxo de informações da modelagem do sistema em tempo real. E ajuda os projetistas a entenderem o funcionamento, mesmo que complexos [9].

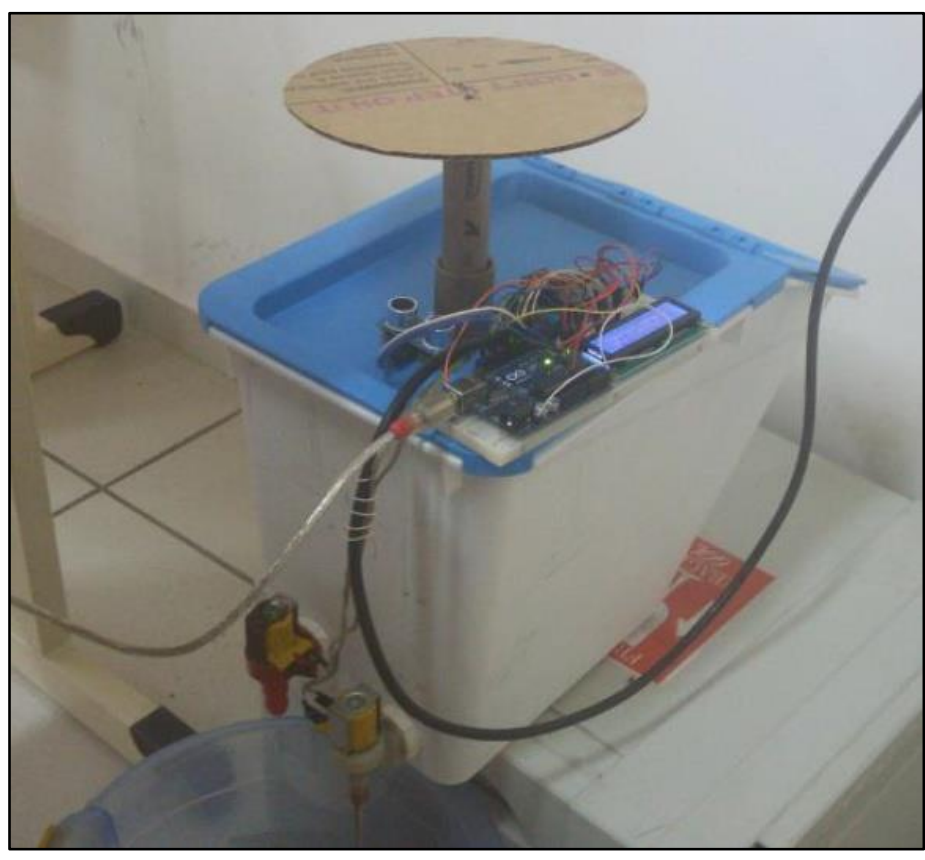

Figura 8: Sistema Montado.

\section{IV.1 DIAGRAMA DE CONTEXTO}

O diagrama de contexto do processo Fig. 9 é composto por fluxo de dados que mostram as interfaces entre o sistema e os componentes externos. No sistema apresentado, o sensor ultrassônico está realizando medições e fornecendo os valores de forma direta ao sistema. O sistema possui duas válvulas solenóides, uma para escoamento de água e outra para escoamento de óleo sendo acionadas pelo Sistema de forma discreta, e um display LCD recebendo sinal discreto do Sistema, fornecendo ao usuário informações sobre medições e mensagens.

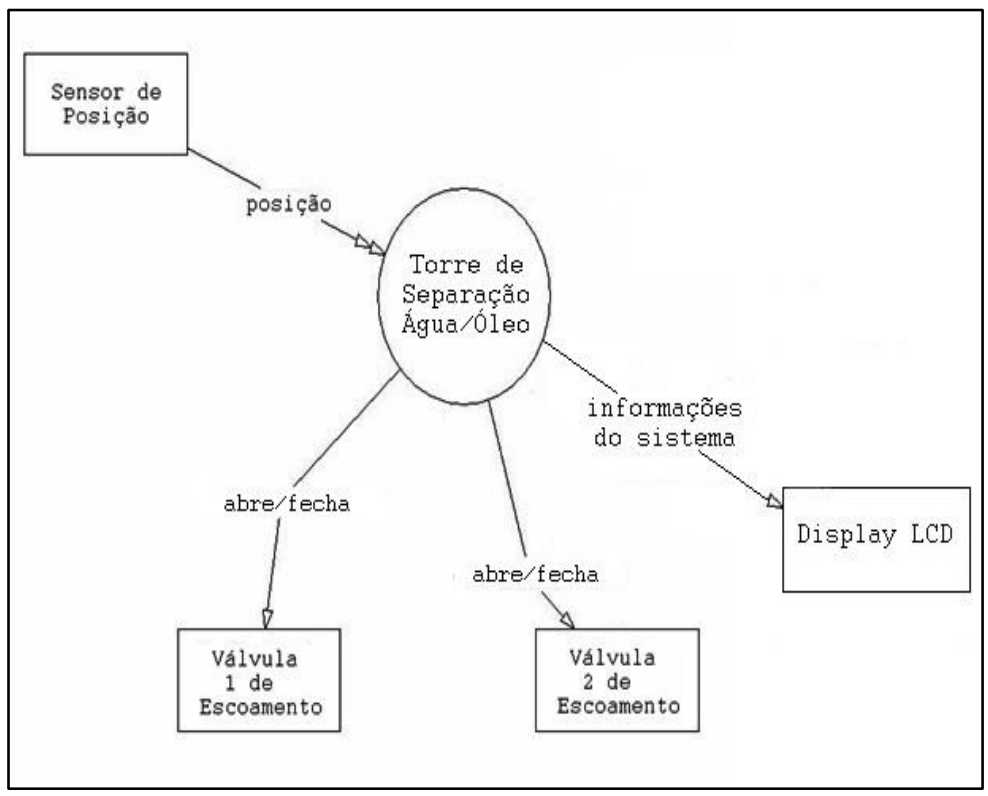

Figura 9: Diagrama de Contexto. 


\section{IV.2 DIAGRAMA DE ESTADOS}

O diagrama de Estados do Sistema Fig. 10 representa o estado em que os objetos do Sistema se encontram no decorrer da execução do processo. Inicialmente o processo está no estado desligado até que o usuário inicie o processo. Se o processo for iniciado e o reservatório possuir líquidos para separação acima do nível mínimo o processo avança para o estado de equipamento ligado, caso contrário retorna ao estado de equipamento desligado.
Quando ligado o Sistema aciona a válvula para escoamento de água permanecendo nesse estado até que o nível de água se aproxime do limite pré-estabelecido no Sistema. Após o escoamento da água o Sistema desliga a válvula da água e liga a válvula para escoamento de óleo, permanecendo neste estado até que escoamento de óleo atinja o nível pré-estabelecido. Assim ocorre o desligamento da válvula do óleo e o Sistema retorna ao estado de equipamento desligado.

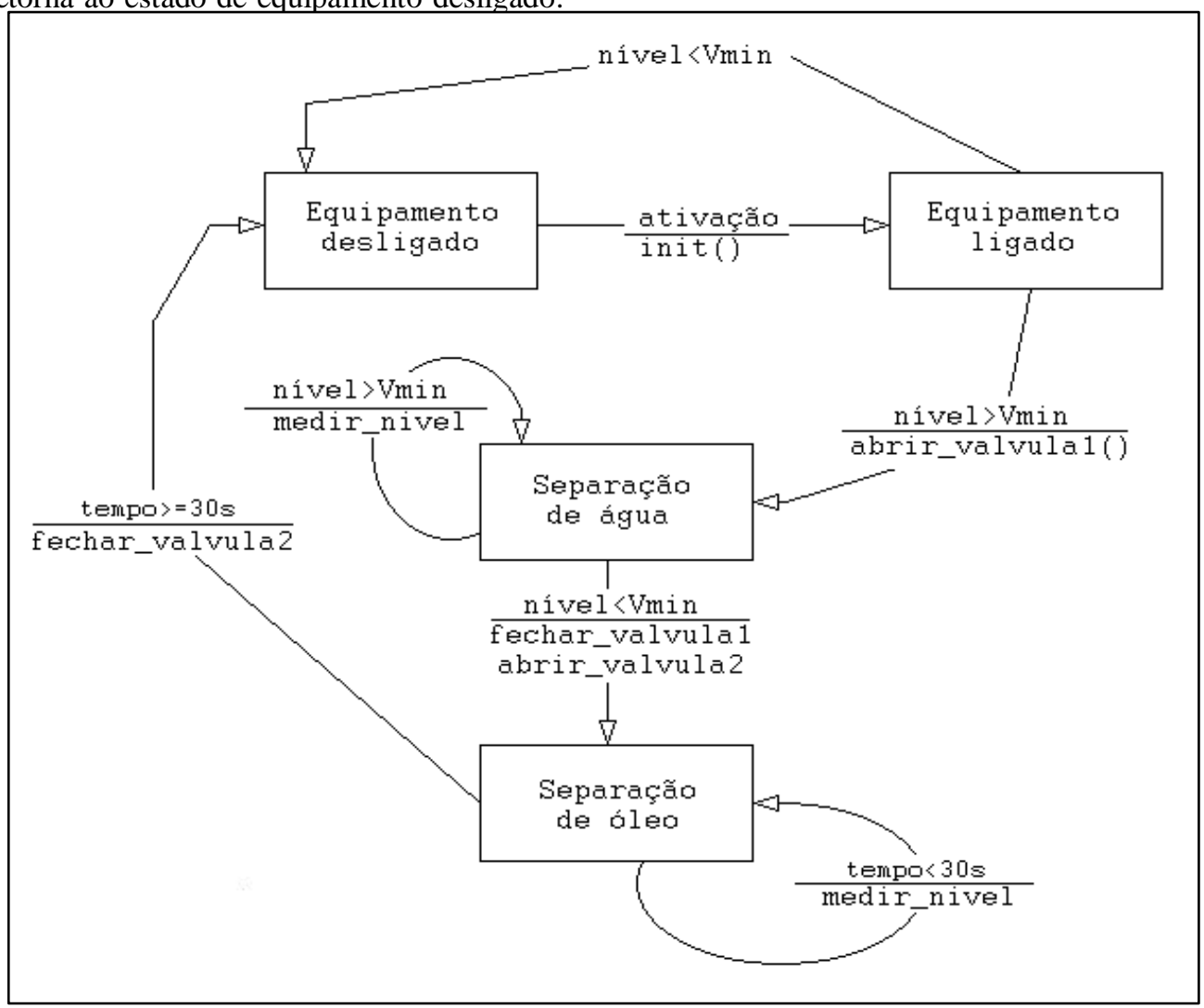

Figura 10: Diagrama de Estados.

\section{METODOLOGIA UML}

$\mathrm{Na}$ segunda etapa de implementação do projeto, foram aplicados conceitos de modelagem de sistemas em tempo real em linguagem UML (Unified Modeling Language). Na aplicação deste conceito foram aplicados diagramas que auxiliam no entendimento do funcionamento do processo.

A metodologia UML também é uma boa ferramenta de comunicação entre as empresas de programação de software para hardware. Os diagramas mostram todas as relações entre as partes do sistema, facilita o uso de banco de dados para uma melhor manutenção e escalabilidade dos equipamentos [10].

\section{V.1 DIAGRAMA DE SEQUÊNCIA}

O diagrama de sequência ilustrado na Figura 11 representa a sequência das mensagens entre operador e os objetos do processo que nesta aplicação são: controle, sensor, válvulas e o display.

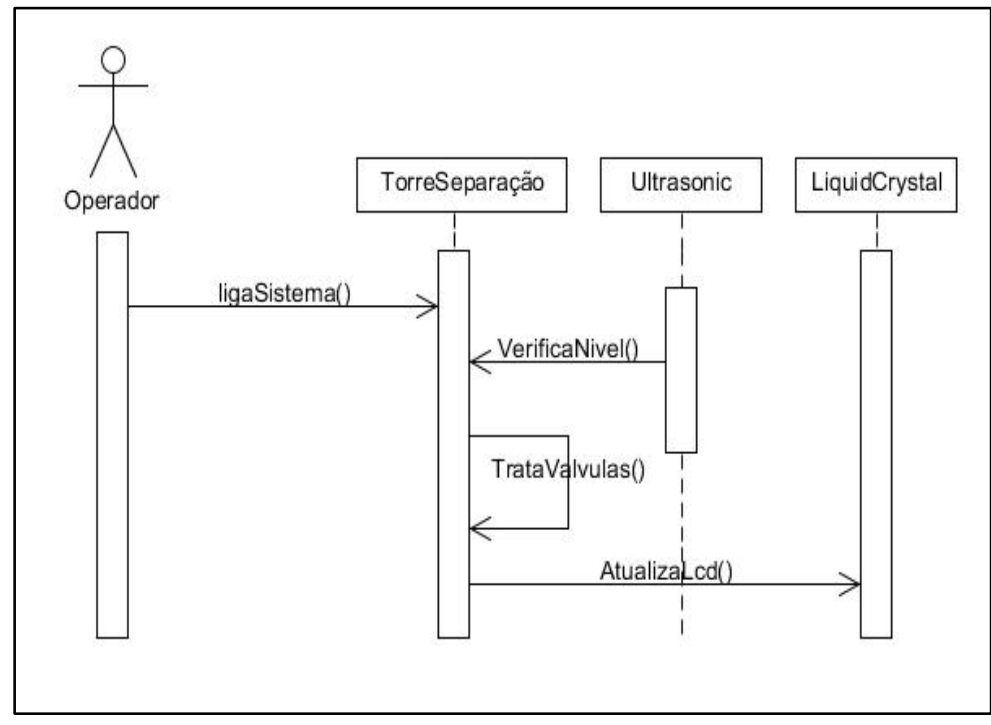

Figura 11: Diagrama de Sequência. 


\section{V.2 DIAGRAMA DE CASOS DE USO}

O diagrama de Casos de Uso representado na Figura 12 tem a finalidade de descrever a sequência dos eventos dos atores que usam o Sistema para completar o processo.

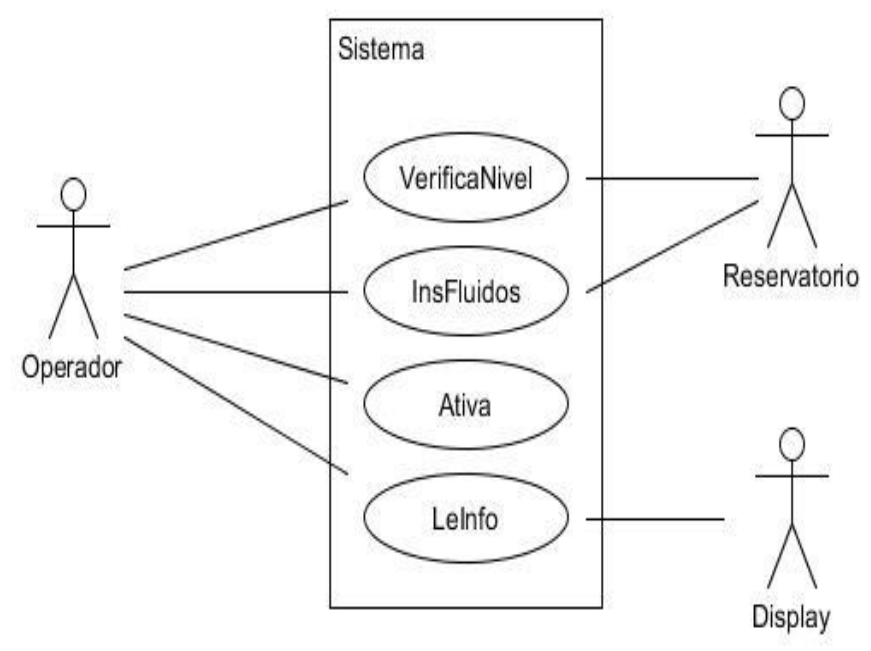

Figura 12: Diagrama de Casos de Uso.

\section{V.3 DIAGRAMA DE CLASSES}

O diagrama de Classes apresentado na figura 13 representa a estrutura e relação de classes que servem de modelo para os objetos da aplicação.

\begin{tabular}{|l|}
\hline \multicolumn{1}{|c|}{ Torre de Separação } \\
\hline Cristal Líquido \\
Ultra-sônico \\
Flutuador cmMsec \\
Tempo Int \\
\hline Parada \\
Laço \\
Nível Verificado \\
Lcd Atualizado \\
Água em \\
Óleo em \\
Válvulas desligadas \\
\hline
\end{tabular}

Figura 13: Diagrama de Classe.

\section{CONCLUSÕES}

O experimento para realizar separação de óleo e água foi desenvolvido com principal objetivo de colaborar com um sistema barato, eficiente e amigável ao usuário para solucionar os problemas encontrados no meio ambiente, principalmente a poluição das águas, e aplicar conceitos teóricos relacionados à disciplina Metodologia de Projeto e Programação de Sistemas de Automação do curso de PósGraduação em Engenharia Elétrica da Universidade Federal do Amazonas - UFAM.

$\mathrm{Na}$ primeira etapa do projeto foram realizados estudos sobre o processo e aplicações, realizando levantamento de materiais e alguns testes com objetivo de verificar se os componentes escolhidos realmente atendem a aplicação.
Através dos conceitos teóricos adquiridos em sala de aula sobre SA-RT, foram apresentados os diagramas de contexto e o diagrama de estados, que definem o funcionamento do Sistema, apontam se os sinais são contínuos ou discretos e definem a ordem do fluxo de informações que deverão ser aplicados posteriormente.

$\mathrm{Na}$ segunda etapa do projeto foram realizadas implementações através dos conceitos da metodologia UML onde foram elaborados os diagramas de sequência, diagrama de casos de uso e diagrama de classes, onde foram definidas informações pertinentes ao fluxo de dados no processo, definição de classes e descrição da sequência de eventos durante o processo.

O sistema mostrou-se adequado para a finalidade que foi montado. A utilização das metodologias resulta na facilidade de reprodução do projeto por um programador e até mesmo de quem possua um conhecimento básico de programação. Como trabalhos futuros, sugere-se que seja acrescentado sensores que façam a leitura do fim do último fluído com a intenção de substituir o tempo para desligar o sistema.

\section{AGRADECIMENTOS}

Ao Programa de Pós-graduação em Engenharia Elétrica da Universidade Federal do Amazonas (PPGEE-UFAM).

\section{REFERÊNCIAS}

[1] Info Escola, Decantação. Disponível em::<http://www.infoescola.com/quimica/decantacao/>. Acesso em 25 de fevereiro de 2017.

[2] Santos, Roberto C. M. M. dos. Estudo de parâmetros relevantes da poluição da água por efluentes de lavanderia $\mathrm{e}$ tinturaria industriais em um rio não perene. Dissertação (Mestrado) - Universidade Católica de Pernambuco. Pró-reitoria Acadêmica. Curso de Mestrado em Desenvolvimento de Processos Ambientais, 2008.

[3] V. C. Barbosa, V.C e Breitschaft, A.M.S. Revista Brasileira de Ensino de Física. Vol.28,p. 110-115. 2006.

[4]P. A. Tripler. Física. Vol.1,ed 5. LTC, Rio de Janeiro, 2000.

[5] J. T. Nascimento. Uso de CFD na Otimização das Proporções Geométricas de um Hidrociclone para a Separação de Dispersões Óleo/Água Provenientes de Refinarias. Rio de Janeiro: UFRJ/EQ, 2008.

[6] M. A. Gomes. Obtenção de biodiesel a partir de resíduos gordurosos obtidos de gorduras anila - vísceras de frango. Escola de Engenharia de Lorena da Universidade de São Paulo, 2010.

[7] Solda Fria, VA-03 | Válvula de entrada de água. Disponível em::<http://www.soldafria.com.br/valvula-solenoide-va03-12-x-12180-graus-24vdc-p-7952.html>. Acesso em 25 de fevereiro de 2017.

[8] Arduino UNO. Disponível em: <https://www.arduino.cc/en/Main/arduinoBoardUno> Acesso em 25 de fevereiro de 2017.

[9] M. N. Lakhoua. Using structured analysis for the control of real-time systems. Journal of Engineering and Technology Research. Vol. 4(5), pp. 82-88, October 2012. 
[10] J. M. Dorador e R. I. M. Young. Application of IDEF0, IDEF3 and UML methodologies in the creation of information models. International Journal of Computer Integrated Manufacturing. Vol 13, 2000. 